\title{
Laboratory Findings in Twins Suffering from
}

\section{Autism-Case Report}

Slobodan Dunjić ${ }^{1}$, Jovana Pejić ${ }^{1}$, Emilija Filipović ${ }^{2}$, Miloš Maletić ${ }^{1}$, Petar Kraković ${ }^{1}$, Dana Stojičić ${ }^{1}$, Milica Lojić $^{1}$ and Vida Subotić ${ }^{3}$

1. Center for Integrative Procedures and Supplements, Belgrade 11070, Serbia

2. Institute for Oncology and Radiology of Serbia, Belgrade 11070, Serbia

3. Zdravlje Pharm, Brčko District 76100, Bosnia and Herzegovina

\begin{abstract}
Autism refers a wide range of conditions and the most prominent are challenges with social skills, repetitive behaviors, speech disturbances. By now, it is known that there is no one type of autism, but there are many of them which are caused by genetic factors and environmental influences. Sometimes autism is called autism spectrum disorders which reflect the wide variation in challenges and strengths possessed by each person with autism (e.g., gastrointestinal disorders, seizures, sleep disturbances, attention deficit and hyperactivity disorders, anxiety etc.). Around one third of all people with autism remain nonverbal and have an intellectual disability. This condition can be detected as early as 18 months, but the most obvious signs tend to appear between 2 and 3 years of age. Only early intervention in those patients can improve outcomes. There are no many articles published regarding laboratory findings in autism patients. By performing holistic approach in patient healing, we also face autistic children in everyday practice. In this paper we represented case of twins suffering from autism. We found that both of them had elevation of free triiodothyronine, oxytocin, osteocalcin, and basophils. We found that vitamin D level in both twins was lower than recommended. Further research in this field is necessary to reveal whether these findings are causal factors for autism or are an effect of the disease.
\end{abstract}

Key words: Autism, osteocalcin, thyroid gland, vitamin D, oxytocin.

\section{Abbreviation}

$\begin{array}{ll}\text { ASD } & \text { Autism spectrum disorder } \\ \text { OT } & \text { Oxytocin } \\ \text { TH } & \text { Thyroid hormones } \\ \text { fT3 } & \text { Free triiodothyronine } \\ \text { FR } \alpha & \text { Folate receptor } \alpha \\ \text { FRAAs } & \text { Folate receptor } \alpha \text { autoantibodies } \\ \text { TPO-Ab } & \text { Thyroid peroxidase antibody } \\ \text { ADHD } & \text { Attention deficit hyperactivity disorder }\end{array}$

\section{Introduction}

Autism spectrum disorder (ASD) is the name for a range of conditions, including Asperger syndrome, that affect a person's social interaction, communication, interests and behavior. The Centers for Disease Control and Prevention (CDC) estimates autism's prevalence

Corresponding author: Slobodan Dunjić, MD, M.Sc., research field: integrative medicine. as 1 in 68 children in the United States. Autism is the most frequent in girls ( 1 in 42 boys versus 1 in 189 girls). When UK is considered, it is estimated that one in every 100 people has autism. Previous means that around 700,000 children in UK live with autism. When Australia is considered, it is estimated that almost 230,000 Australians live with ASD which is around four times more frequent in boys than in girls. Surprisingly, India, one of the biggest countries in the world, has no accurate data of autism prevalence [1]. Children having autism express symptoms before the age of three. Diagnosis can sometimes be made by the age of three or later. Only early intervention in those patients can improve outcomes.

Causes of ASD are various; one of them is for sure gene influence. However, no specific genes are linked with ASD. Aside of genes, certain influence can be attributed to environmental triggers. The most 
suspected environmental compounds listed in epidemiology that can have influence on ASD, are tetrachlorodibenzodioxin, pesticides, benzo(a)pyrene, heavy metals, valproate, acetaminophen, cocaine, bisphenol A, phthalates, polyhalogenated biphenyls, diesel constituents, etc. Also, there are listed endocrine disruptors (over 100) including paraquat, atrazine and other pesticides not yet studied in autism and many compounds used in food, cosmetics or household products (including tretinoin, soy phytoestrogens, aspartame, titanium dioxide, and sodium fluoride). Recent studies have shown that heavy exposure to pesticides and air pollution (especially particulate matter $<2.5$ and $10 \mu \mathrm{m}$ in diameter) during pregnancy is also associated with ASD [2]. Many polluting chemical substances can interfere with thyroid hormone (TH) metabolism and this might lead to abnormalities in the neurological development of the fetus or the child. Some papers describe connection between thyroid gland dysfunction and autism development [3]. Researchers also examined other possible related factors. They found that maternal infections (rubella and CMV with fetal brain injuries, and possibly Influenza with fever), prolonged fever and maternal inflammation, especially with changes in a variety of inflammatory cytokines and antibodies that cross the placenta can lead to ASD by affecting the fetal brain. There are also drugs which can be related to ASD development. Following drugs are listed: valproic acid, thalidomide, misoprostol, etc. $\beta 2$-adrenergic receptor agonists and paracetamol have also lately been associated with increased rate of ASD but the data are too preliminary and inconclusive [4]. Advanced parental age is also associated with higher risk of ASD. Birth complications that are associated with trauma or ischemia and hypoxia have also shown strong links to ASD. On the other side, other pregnancy-related factors such as maternal obesity and diabetes, and caesarian section have shown a less strong (but significant) association with risk of ASD. Food allergies are often associated with ASD. In one case-report, milk and peanut allergies were reported in 13 months old child [5]. There is growing interest in the role of the oxytocin system in social cognition and behavior. The neuropeptide oxytocin and its receptor have been predicted to be involved in the regulation of social functioning in autism spectrum disorders. Some researchers investigated the level of oxytocin (OT) in people suffering from ASD. They found that individuals with ASD may exhibit a dysregulation in OT on the basis of changes in OT receptor gene expression [6]. OT can be released from brain during stressful condition [7]. On the other side, there is an increased interest in research of possible role of vitamin D in autism people. So far, vitamin D has been known as relevant factor for bone health. However, evidence has recently accumulated for an impact of vitamin D on mental health. Namely, results from 25 cross-sectional studies, as well as, from 8 longitudinal studies suggest a role of vitamin $\mathrm{D}$ in the pathogenesis of mental disorders in childhood and adolescence [8]. Also, many studies have highlighted hypovitaminosis-D as a potential environmental risk factor for a variety of conditions such as multiple sclerosis, asthma, cardiovascular disease, and, more recently, psychiatric diseases. However, whether hypovitaminosis-D is a potential causative factor for the development or activity in these conditions or whether hypovitaminosis-D may be due to increased vitamin-D consumption by an activated immune system (reverse causation) is the focus of intense research [9]. Usually, diagnosis goes thought two stages-one is general developmental screening during well-child checkups, and the second is additional evaluation which is done by different specialists, such are, psychologist, psychiatrists, pediatrician, speech and language therapist. However, no much data are published regarding laboratory findings in people suffering from ASD. Most used therapies for patient suffering from ASD are speech and language therapy, occupational therapy, educational support etc. As for medication, there are no specific therapies but the easy some problems such 
Table 1 Values of biochemical parameters which were out of physiological range in twins suffering from ASD.

\begin{tabular}{llllll}
\hline $\begin{array}{l}\text { Parameter (unit and } \\
\text { physiological range) }\end{array}$ & $\begin{array}{l}\mathrm{fT3} \\
\text { (pmol/L; 3,1-6,8) }\end{array}$ & $\begin{array}{l}\text { Vitamin D } \\
\text { (nmol/L; 75-200) }\end{array}$ & $\begin{array}{l}\text { Oxytocin } \\
(\mathrm{pg} / \mathrm{mL} ; \text { 40-210) }\end{array}$ & $\begin{array}{l}\text { Osteocalcin } \\
\text { (ng/mL; 24-70) }\end{array}$ & $\begin{array}{l}\text { Monocites } \\
(\% ; 3.0-8.0 \%)\end{array}$ \\
\hline One brother & 6.98 & 41.3 & 536.8 & 78 & 8.4 \\
\hline Other brother & 7.06 & 30.7 & 394.4 & 74.9 & 10.6 \\
\hline
\end{tabular}

are irritability, aggression, repetitive behavior, hyperactivity etc. [10-12].

\section{Case Report}

The paper is going to present laboratory data we found in twins suffering from ASD. Brothers are 4 years of age. They have been diagnosed with ASD and came for additional holistic treatment in Center for Integrative procedures and Supplements "Dr Dunjić, Belgrade, Serbia. Staff of the Center deal with holistic approach in treating the patients and the therapy is fully personalized (every person receives personally tailored treatment). Approach in healing the patients is not to treat disease/condition, but to treat whole body trying to resolve homeostasis which is impaired. Following check-up, boys were referred to standard laboratory analysis. The vast majority of the biochemical analyses were within physiological range. In this paper, only specific analyses which were out of physiological values will be presented. The data are shown in Table 1.

\section{Discussion}

Results from previous table show that the level of free triiodothyronine (fT3) was higher than maximal proposed level in both twins. There are numerous papers discussing possible connection between thyroid gland disfunction and ASD. Thus in one paper, author noted that folate receptor $\alpha(\mathrm{FR} \alpha)$ auto antibodies (FRAAs) are prevalent in autism spectrum disorder. The main mechanism of action would be that FRAAs disrupt folate transport across the blood-brain barrier by binding to the FR $\alpha$. Thyroid dysfunction is frequently found in children with ASD. Also, paper stated that foetal and neonatal exposure to maternal FRAAs could affect the development of the thyroid and may contribute to the pathology in ASD [13]. There is a research which tried to find connection between autoimmune thyroiditis and ASD. Namely, researcher followed mothers with autoimmune thyroiditis having elevation of thyroid peroxidase antibody during pregnancy (TPO-Ab; elevation is considered if $>156 \mathrm{IU} / \mathrm{mL}$ ) and related that finding with and development of ASD in their children. Study was conducted in Finland. There were 967 matched case-control pairs enrolled in this study, and data were analyzed by conditional logistic regression for matched sets. Researchers found that the prevalence of maternal TPO-Ab+ was significantly increased in pregnancies giving rise to autism cases (6.15\%) compared to controls (3.54\%). Also, the odds of autism were increased by nearly $80 \%$ among offspring of mothers who were TPO-Ab+ during pregnancy $(\mathrm{OR}=1.78,95 \% \mathrm{CI}=1.16-2.75, p=0.009)$, compared to mothers negative for this autoantibody. There was also a significant relationship between maternal TPO-Ab defined as a continuous variable and odds of autism (OR $=1.09,95 \% \mathrm{CI}=1.01,1.17$, $p=0.02)$. Finally, authors concluded that autoimmune disorders are related to autism in offspring [14].

Very interesting results came out from Danish study which is done between 1991 and 2004. The study enrolled 857,014 singletons alive and living in Denmark at the age of 3 years. The Cox proportional hazards model was applied to estimate the hazard ratio (HR) with 95\% confidence interval (95\% CI) for risk of ASD in children born to mothers with thyroid dysfunction. Analysis was adjusted for potential confounding factors. Altogether, 30,295 singletons (3.5\%) were born to mothers with thyroid dysfunction. Both maternal hyperthyroidism diagnosed and treated for the first time after the birth of the child and 
hypothyroidism increased the risk of ASD in the child (adjusted HR 1.23; 95\% CI 1.05-1.44; adjusted HR 1.34; 95\% CI 1.14-1.59). Authors explained this finding by the fact that children may have been exposed to abnormal levels of maternal thyroid hormone already present during the pregnancy, and this untreated condition could increase the risk of specific neurodevelopmental disorders in those children [15]. In the focus of recent researches is also possible association between deficiency of some vitamins and ASD development. In one study, there were 77 children and adolescents of both sexes enrolled. 50 of them had attention deficit hyperactivity disorder (ADHD) and ASD. The rest of studied group ( $n=27$ ) was their control. The blood samples were obtained between 8:00 and 9:00 A.M. The ASD group had the highest ferritin and the lowest vitamins B12 and D levels. Vitamin D levels of the ADHD group were significantly lower compared to the healthy controls. Based on this study results, authors concluded that there is a need for supplementation of vitamins $\mathrm{B} 12$ and $\mathrm{D}$ in the ASD and ADHD patients [16]. Also, deficiency of vitamin D in ASD patients was seen in other studies and the importance for supplementation of this vitamin is highlighted [17].

When vitamin D is considered, it was found that the level in both twins was lower than it is recommended. Now, it is very good known that vitamin $\mathrm{D}$ is lower in children with ASD. Vitamin D, as hormone, regulates about $3 \%$ of the 26,000 genes in the coding human genome. It is found that vitamin $\mathrm{D}$ also regulates brain development and has an effect on neurotransmission and synaptic plasticity. Some studies found that high dose vitamin D improves the core symptoms of autism in about $75 \%$ of autistic children. The doses used in some studies were $400 \mathrm{IU} / \mathrm{day}, 5,000 \mathrm{IU} /$ day up to $150,000 \mathrm{IU} / \mathrm{month} \mathrm{IM}$. On the other hand, in terms of prevention, one study showed that supplementation during pregnancy with 5,000 IU/day and during infancy and early childhood (1,000 IU/day) significantly reduced the expected incidence of autism in mothers who already had one autistic child from 5\% to $20 \%$ [18]. Also, animal studies have suggested that transient prenatal vitamin D deficiency is associated with altered brain development [19].

The development of ASD is suggested to be related to abnormalities of the oxytocin-arginin-vasopressin system. Previous results suggest that OXT and arginine vasopressin (AVP) may play a role in the etiopathogenesis of ASD [20]. The effects of oxytocin on social cognition and behavior have recently attracted considerable attention and numerous papers have researched the possible role of oxytocin as therapeutic agent to treat ASD [21, 22]. Animal models also demonstrated that the neuropeptide oxytocin plays an important role in social recognition and bonding. On the bonding, human-based research has explored its therapeutic potential for social dysfunction in psychiatric disorders [23]. The results that we obtained in our research are somehow controversial in relation to current attitude regarding the use of oxytocin and ASD treatment. At one side we found an elevation of oxytocin level and, at the other, review of literature showed that oxytocin is promising therapeutic agent in treating an ASD. The authors of the paper would like to discuss that this result could be due to high presence of less active oxytocin and/or presence of less sensitive receptors for oxytocin.

By searching a medical literature, no much data were found regarding osteocalcin and development of ASD. One interesting research was done by Karsenty and associates. They showed that osteocalcin crosses the placenta from mother to fetus and that this maternal pool of osteocalcin is necessary for formation of the hippocampus and the establishment of memory. Lastly, they showed that once-a-day injections of osteocalcin in osteocalcin-null mothers during pregnancy could prevent the development of behavioral abnormalities in their offspring. The study was done on mice. The researchers showed unambiguously that osteocalcin can cross the 
blood-brain barrier and bind to neurons in the brainstem, midbrain, and hippocampus (which is responsible for learning and memory). Osteocalcin promotes the birth of neurons and increases the synthesis of several neurotransmitters, including serotonin, dopamine, and catecholamine. They also found that osteocalcin-null mice had abnormally small hippocampi [24]. In these twins with ASD we found an elevation of osteocalcin level. Based on scarced literature published so far, one may expect that osteocalcin level in children suffering from ASD would be lower than in children with no ASD. However, such result was not obtained. Possible explanation for this finding is that osteocalcin was less effective in these two subjects which resulted in elevation of its plasma level. The other possible reason for this finding could be presence of less sensitive receptors for osteocalcin which, in turn, caused elevation of osteocalcin. Aside of this finding, we observed that vast majority of our patients-children with ASD have an elevation of osteocalcin. However, no statistical analysis was done so far to compare mean levels of osteocalcin between children having ASD and their controls.

Finally we observed an elevation of basophils in differential blood count. Basophils play an important role in preventing blood clotting; this is due to heparin that they contain. Also, thanking to heparin, they have an important role in allergic reactions, and they help body to produce antibodies called immunoglobulin $\mathrm{E}$ (IgE). Plenty of different conditions can cause an elevation of basophils in blood stream, such are viral infections, lymphoma, hemolytic anemia, following splenectomy, myeloproliferative diseases, inflammation, endocrine diseases and conditions. However, to date we found no study describing an elevation of basophils in autistic children. According to our observation based on every day practice, this finding could be explained by inappropriate reaction of immune system in those children which, very often, have allergic reactions. These twins have allergies in anamnesis.

\section{Conclusions}

By presentation of these two cases of twin brothers with ASD, we found an elevation of oxytocin, osteocalcin, basophils, fT3, and decrease in vitamin D level. The associations described in this review emphasize again how little we know about the etiology and pathogenesis of ASD. According to our observation from everyday practice, we have noticed that vast majority of autism patients have elevation of osteocalcin, oxytocin, and presence of impaired function of thyroid gland. However, we did not perform statistical analysis to test if the difference is statistically significant in comparison to controls. It is obvious that we need more epidemiologic data to establish many of these associations, but if proven, they might be promising avenues for prevention and treating ASD.

\section{Conflict of Interest}

No any financial interest or conflict of interest exists.

\section{References}

[1] Rudra, A., Belmonte, M. K., Soni, P. K., Banerjee, S., Mukerji, S., and Chakrabarti, B. 2017. "Prevalence of Autism Spectrum Disorder and Autistic Symptoms in a School-based Cohort of Children in Kolkata." Autism Res. 10 (10): 1597-605. doi: 10.1002/aur.1812. Epub 2017 May

https://www.ncbi.nlm.nih.gov/pubmed/28544637.

[2] Carter, C. J., and Blizard, R. A. 2016. “Autism Genes Are Selectively Targeted by Environmental Pollutants Including Pesticides, Heavy Metals, Bisphenol A, Phthalates and Many Others in Food, Cosmetics or Household Products.” Neurochem S0197-0186 (16): 30197-8. doi: 10.1016/j.neuint.2016.10.011. [Epub ahead of print]. https://www.ncbi.nlm.nih.gov/pubmed/?term=2.\%09Cart er+CJ\%2C+Blizard+RA.+Autism+genes+are.

[3] Ozzola, G. 2016. "Pollution, the Thyroid and Neurodevelopment.” Clin Te. 167 (6): 191-7. doi: 10.7417/CT.2016.1972.

https://www.ncbi.nlm.nih.gov/pubmed/?term=3.\%09Ozz ola+G1.+Pollution\%2C+the+thyroid+and.

[4] Ornoy, A., Weinstein-Fudim, L., and Ergaz, Z. 2016. 
“Genetic Syndromes, Maternal Diseases and Antenatal Factors Associated with Autism Spectrum Disorders (ASD)." Front Neurosci 10: 316. doi: 10.3389/fnins.2016.00316. eCollection 2016. https://www.ncbi.nlm.nih.gov/pubmed/27458336.

[5] Lucarelli, J., Pappas, D., Welchons, L., and Augustyn, M. 2017. "Autism Spectrum Disorder and Avoidant/Restrictive Food Intake Disorder.” J Dev Behav $\begin{array}{llll}\text { Pediatr. } & 38 & \text { (1): } & \text { 79-80. }\end{array}$ 10.1097/DBP.0000000000000362.

https://www.ncbi.nlm.nih.gov/pubmed/27824638.

[6] Yang, S., Dong, X., Guo, X., Han, Y., Song, H., Gao, L. Dai, W., Su, Y., and Zhang, X. 2017. "Serum Oxytocin Levels and an Oxytocin Receptor Gene Polymorphism (rs2254298) Indicate Social Deficits in Children and Adolescents with Autism Spectrum Disorders.” Front Neurosci 11: 221. doi: 10.3389/fnins.2017.00221. eCollection 2017. https://www.frontiersin.org/articles/10.3389/fnins.2017.0 0221/full.

[7] Valstad, M., Alvares, G. A., Egknud, M., Matziorinis, A. M., Andreassen O. A., Westlye L. T., and Quintana D. S. 2017. "The Correlation between Central and Peripheral Oxytocin Concentrations: A Systematic Review and Meta-analysis.” Neurosci Biobehav Rev. 78: 117-24. doi: 10.1016/j.neubiorev.2017.04.017. [Epub ahead of print]. https://www.ncbi.nlm.nih.gov/pubmed/28442403.

[8] Föcker, M., Antel, J., Ring, S., Hahn, D., Kanal, Ö., Öztürk, D., Hebebrand, J., and Libuda, L. 2017. "Vitamin D and Mental Health in Children and Adolescents.” Eur Child Adolesc Psychiatry doi: 10.1007/s00787-017-0949-3. [Epub ahead of print]. https://www.ncbi.nlm.nih.gov/pubmed/?term=Vitamin+D +and+mental+health+in+children+and+adolescents.+Eur +Child+Adolesc+Psychiatry. $+2017+$ Feb+8.+doi\%3A+10 .1.

[9] Kočovská, E., Gaughran, F., Krivoy, A., and Meier, U. C. 2017. "Vitamin-D Deficiency as a Potential Environmental Risk Factor in Multiple Sclerosis, Schizophrenia, and Autism.” Front Psychiatry 8: 47. doi: 10.3389/fpsyt.2017.00047. eCollection 2017. https://www.ncbi.nlm.nih.gov/pmc/articles/PMC5366333 I.

[10] https://www.nimh.nih.gov/health/topics/autism-spectrumdisorders-asd/index.shtml?utm_source=rss_readersutm_ medium=rssutm_campaign=rss_full.

[11] http://www.autism.org.uk/about/what-is/asd.aspx.

[12] https://www.autismspectrum.org.au/content/what-autism.

[13] Frye, R. E., Wynne, R., Rose, S., Slattery, J., Delhey, L., Tippett, M., Kahler, S. G., Bennuri, S. C., Melnyk, S., Sequeira, J. M., and Quadros, E. V. 2017. “Thyroid Dysfunction in Children with Autism Spectrum Disorder
Is Associated with Folate Receptor $\alpha$ Autoimmune Disorder.” J Neuroendocrinol 29 (3). doi: 10.1111/jne.12461.

https://www.ncbi.nlm.nih.gov/pubmed/28199771.

[14] Brown, A. S., Surcel, H. M., Hinkka-Yli-Salomäki, S., Cheslack-Postava, K., Bao, Y., and Sourander, A. 2015. "Maternal Thyroid Autoantibody and Elevated Risk of Autism in a National Birth Cohort." Prog Neuropsychopharmacol Biol Psychiatry 57: 86-92. doi: 10.1016/j.pnpbp.2014.10.010. Epub 2014 Oct 29. https://www.ncbi.nlm.nih.gov/pubmed/25445476.

[15] Andersen, S. L., Laurberg, P., Wu, C. S., and Olsen, J. 2014. “Attention Deficit Hyperactivity Disorder and Autism Spectrum Disorder in Children Born to Mothers with Thyroid Dysfunction: A Danish Nationwide Cohort Study.” BJOG 121 (11): 1365-74. doi: 10.1111/1471-0528.12681. Epub 2014 Mar 10. https://www.ncbi.nlm.nih.gov/pubmed/24605987.

[16] Bala, K. A., Doğan, M., Kaba, S., Mutluer, T., Aslan, O., and Doğan, S. Z. 2016. "Hormone Disorder and Vitamin Deficiency in Attention Deficit Hyperactivity Disorder (ADHD) and Autism Spectrum Disorders (ASDs).” $J$ Pediatr Endocrinol Metab 29 (9): 1077-82. doi: 10.1515/jpem-2015-0473.

https://www.ncbi.nlm.nih.gov/pubmed/27544717.

[17] Modabbernia, A., Velthorst, E., and Reichenberg, A. 2017. "Environmental Risk Factors for Autism: An Evidence-based Review of Systematic Reviews and Meta-analyses.” Mol Autism 8: 13. doi: 10.1186/s13229-017-0121-4. eCollection 2017. https://www.ncbi.nlm.nih.gov/pubmed/28331572.

[18] Cannell, J. J. 2017. "Vitamin D and Autism, What's New?" Rev Endocr Metab Disord. doi: 10.1007/s11154-017-9409-0. [Epub ahead of print] https://www.ncbi.nlm.nih.gov/pubmed/28217829.

[19] Ali, A., Cui, X., and Eyles, D. 2016. "Developmental Vitamin D Deficiency and Autism: Putative Pathogenic Mechanisms.” J Steroid Biochem Mol Biol S0960-0760 (16): 30365-X. doi: 10.1016/j.jsbmb.2016.12.018. [Epub ahead of print]. https://www.ncbi.nlm.nih.gov/pubmed/28027915.

[20] Vanya, M., Szucs, S., Vetro, A., and Bartfai, G. 2017. "The Potential Role of Oxytocin and Perinatal Factors in the Pathogenesis of Autism Spectrum Disorders-Review of the Literature." Psychiatry Res 247: 288-90. doi: 10.1016/j.psychres.2016.12.007. Epub 2016 Dec 6. https://www.ncbi.nlm.nih.gov/pubmed/27974283.

[21] Benner, S., and Yamasue, H. 2017. “Clinical Potential of Oxytocin in Autism Spectrum Disorder: Current Issues and Future Perspectives.” Behav Pharmacol. doi: 10.1097/FBP.0000000000000341. [Epub ahead of print]. https://www.ncbi.nlm.nih.gov/pubmed/28857771. 
[22] Wagner, S., and Harony-Nicolas, H. 2017. "Oxytocin and Animal Models for Autism Spectrum Disorder.” Curr Top Behav Neurosci. doi: 10.1007/7854_2017_15. [Epub ahead

of print]. https://www.ncbi.nlm.nih.gov/pubmed/28864977.

[23] Kendrick, K. M., Guastella, A. J., and Becker, B. 2017. "Overview of Human Oxytocin Research.” Curr Top Behav Neurosci. doi: 10.1007/7854_2017_19. [Epub ahead print]. https://www.ncbi.nlm.nih.gov/pubmed/28864976.

[24] Oury, F., Khrimian, L., Denny, C. A., Gardin, A., Chamouni, A., Goeden, N., Huang, Y.-Y., Lee, H., Srinivas, P., Gao, X.-B., Suyama, S., Langer, T., Mann, J. J., Horvath, T. L., Bonnin, A., and Karsenty, G. 2013. "Maternal and Offspring Pools of Osteocalcin Influence Brain Development and Functions.” Cell 155 (1): 228 DOI: 10.1016/j.cell.2013.08.042. https://www.ncbi.nlm.nih.gov/pubmed/24074871. 